\title{
New technique for real-time interface pressure analysis: Getting more out of large image data sets
}

\author{
Kath Bogie, DPhil; ${ }^{1-2^{*}}$ Xiaofeng Wang, PhD; ${ }^{3-4}$ Baowei Fei, PhD; ${ }^{5}$ Jiayang Sun, PhD $^{6}$ \\ ${ }^{1}$ Cleveland Advanced Platform Technology Center, Louis Stokes Cleveland Department of Veterans Affairs Medical \\ Center, Cleveland, $\mathrm{OH} ;{ }^{2}$ Department of Orthopaedics, Case Western Reserve University, Cleveland, OH; ${ }^{3}$ Department \\ of Quantitative Health Sciences, The Cleveland Clinic, Cleveland, $\mathrm{OH} ;{ }^{4}$ Cleveland Clinic Lerner College of Medicine, \\ Case Western Reserve University, Cleveland, $\mathrm{OH} ;{ }^{5}$ Departments of Radiology and Biomedical Engineering, Case \\ Western Reserve University and University Hospitals Case Medical Center, Cleveland, OH; ${ }^{6}$ Department of Statistics, \\ Case Western Reserve University, Cleveland, $\mathrm{OH}$
}

\begin{abstract}
Recent technological improvements have led to increasing clinical use of interface pressure mapping for seating pressure evaluation, which often requires repeated assessments. However, clinical conditions cannot be controlled as closely as research settings, thereby creating challenges to statistical analysis of data. A multistage longitudinal analysis and self-registration (LASR) technique is introduced that emphasizes real-time interface pressure image analysis in three dimensions. Suitable for use in clinical settings, LASR is composed of several modern statistical components, including a segmentation method. The robustness of our segmentation method is also shown. Application of LASR to analysis of data from neuromuscular electrical stimulation (NMES) experiments confirms that NMES improves static seating pressure distributions in the sacral-ischial region over time. Dynamic NMES also improves weight-shifting over time. These changes may reduce the risk of pressure ulcer development.
\end{abstract}

Key words: biomedical imaging, gluteal stimulation, LASR, longitudinal analysis and self-registration, interface pressure mapping, neuromuscular electrical stimulation, pressure ulcers, real-time statistical analysis, rehabilitation, seating pressure, self-registration, translational research.

\section{INTRODUCTION}

Interface pressure mapping has become an increasingly widely used assessment tool in wheelchair and seat- ing clinics [1-2]. It is often used for clients with complex seating needs as part of the evaluation procedure for customized seating, including pressure-relieving cushions. Such clients often require repeated evaluations, either longitudinally or as an objective measure in the evaluation of posture and function on different seating systems. Before its adoption in routine clinical use, interface pressure distribution was used primarily in research applications, such as our study, to determine the effects of neuromuscular electrical stimulation (NMES) on pressure ulcer prevention [3].

The move from research applications to clinical utility has largely been due to manufacture-led improvements in

Abbreviations: $2-\mathrm{D}=$ two-dimensional, $3-\mathrm{D}=$ threedimensional, $\mathrm{CT}=$ computed tomography, $\mathrm{fMRI}=$ functional magnetic resonance imaging, $\mathrm{FDR}=$ false-discovery rate, i.i.d $=$ independent and identically distributed, $\mathrm{L}=$ left, LASR $=$ longitudinal analysis and self-registration, NMES = neuromuscular electrical stimulation, $\mathrm{R}=$ right, $\mathrm{SCI}=$ spinal cord injury, $\mathrm{SVD}=$ singular value decomposition.

*Address all correspondence to Kath Bogie, DPhil; Cleveland Advanced Platform Technology Center, Louis Stokes Cleveland VA Medical Center, 10701 East Boulevard, Cleveland, OH 44106; 216-368-5270; fax: 216-778-4259. Email: kmb3@case.edu

DOI: 10.1682/JRRD.2007.03.0046 
both hardware and software: the design criteria for the ideal pressure sensor system have been defined [4]. Sensors have become more reliable and calibration procedures have become more robust. More intuitive software programs both increase the usability for the nonexpert operator and allow real-time feedback on changing conditions at the seating support interface. However, no single device meets all the design criteria nor has one been identified as the gold standard for interface pressure measurement. Furthermore, techniques for the quantitative analysis of pressure mapping data have not kept pace with product development. Advanced analytical methods have been reported for specific applications in research studies. For example, Brienza et al. used a data reduction and analysis technique based on singular value decomposition (SVD) to compare interface pressure maps and shape contour maps for seated subjects [5]. In this case, the goal was to establish overall similarities between different pressure measurements (via SVD). Aissaoui et al. described a deformable active contour-segmentation method that analyzed the pressure distribution at the user-defined ischial region of the body-seat interface in nondisabled subjects and subjects with paraplegia [6]. The goal of their study was to estimate the regional surface and supported load of seated nondisabled individuals and individuals with spinal cord injury (SCI). In order to enhance understanding of the body structural factors that contribute to peak pressure, Hastings et al. developed a registration technique to align X-ray computed tomography (CT) data with pressure sensor data [7]. Our goal was to employ an automatic approach with no observerselected region of interest. We further wished to conduct clinical assessments when simultaneous differences between seating cushions (i.e., for the entire spatial seating region) were the primary outcome of interest. This approach is analogous to an experienced radiologist evaluating a patient using multiple images simultaneously. Thus, our approach seeks to determine the significance of differences in all pixel locations simultaneously (i.e., pixel by pixel for all pixels simultaneously and subject by subject).

Our goal cannot be achieved by only a snapshot or by a small number of numerical values (e.g., mean and maximum). As stated in Stinson et al., "Anecdotal evidence suggests that health care professionals tend to use the pressure maps more than the numerical values" [8]. The authors reported good agreement between maximum pressure values and evaluations based on subjective ranking of pressure maps from "best" to "poorest," particularly when the seating surfaces being compared were dissimilar, e.g., hard seating surface and foam/gel cushion. However, average pressures and subjective rankings of similar seating surfaces did not show good agreement. Single-frame pressure maps were used for comparison and evaluation, thus no measure of change over time was considered. This type of approach can be considered analogous to determining inner-city traffic patterns from a single $30,000 \mathrm{ft}$ aerial image; an overall impression at one moment may be obtained but significant dynamic data are lost and no longitudinal information regarding change over time is feasible. Another example can be found in the typical pressure maps depicted in Figure 1; comparison of the mean interface pressure values would imply that Figure 1(b) is a "better" pressure distribution because the mean pressure is lower. Visual comparison shows clearly that Figure 1(a) is more homogeneous, i.e., regional interface pressures are more evenly distributed. Including the maximum pressure gives some further information, but even so, regions of critically changing pressure distribution still cannot be specified with the information from the maximum and mean values of an image alone.

A different analytical methodology for quantitative assessment of change over time or under different conditions for the full pressure map is needed for enhanced clinical evaluation of interface pressure distribution. In the clinical setting, the different conditions can include repeated short-term assessments such as assessing a client for three different seating cushions or evaluating the same seating system in different configurations, e.g., tiltin-space or with footrest adjustment. Clients are also often seen on more than one occasion over a period of months or years, and the ability to register maps/images to make valid comparisons between pressure maps obtained over long intervals is important. In either situation, clinical interpretation is most efficient if pressure maps can be analyzed in a real-time or near real-time manner. As defined further in the "Description of LASR Algorithm” section, p. 526, pressure mapping produces large volume data sets. As a result, the ability to provide real-time analysis has been limited because of the extended processing times required.

This article presents an analytical technique, longitudinal analysis and self-registration (LASR), that determines statistically valid changes between baseline and posttreatment pressure maps obtained over both the short- and long-term. The LASR algorithm employs data 
(a)

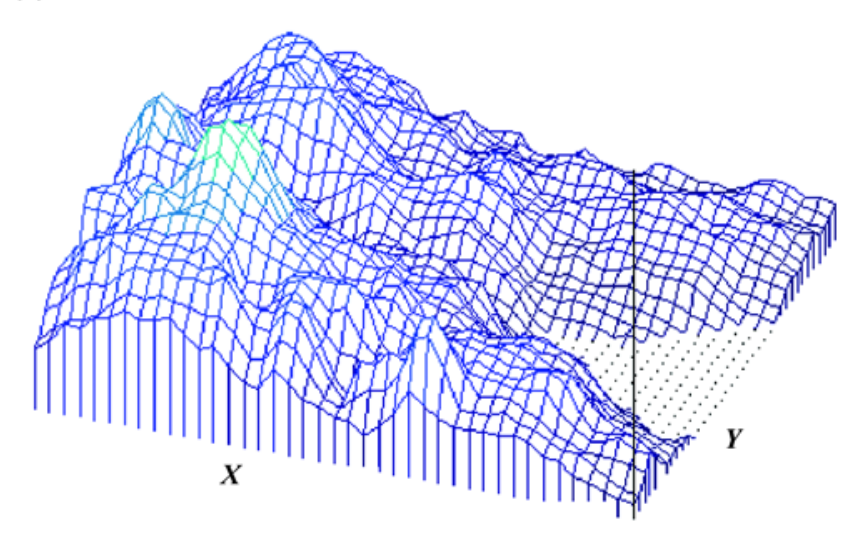

(b)

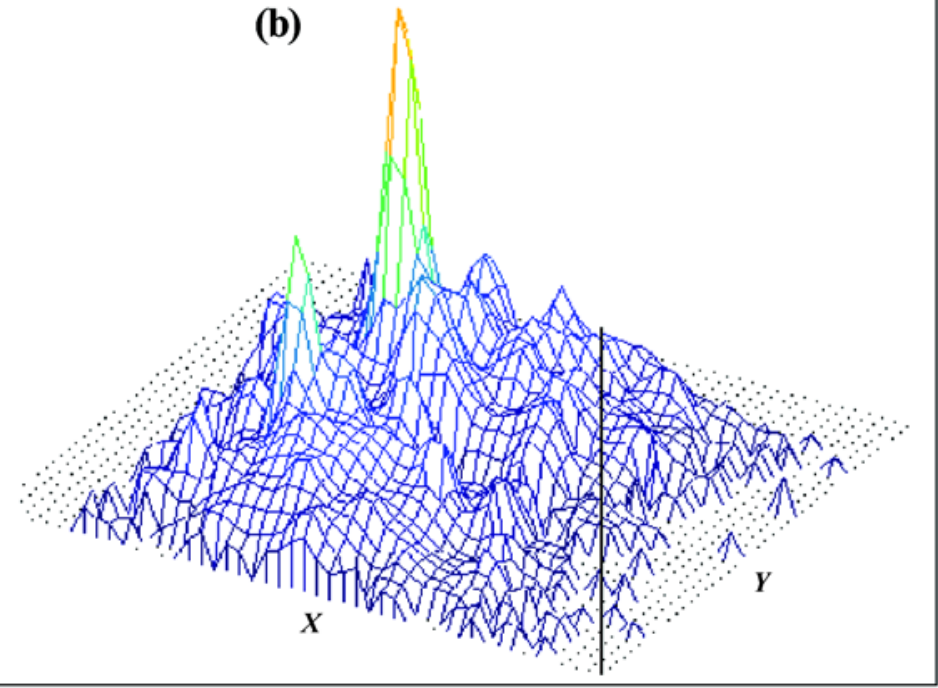

\section{\begin{tabular}{l|l|l|l|l|l|l|l|l|l|l|l}
127 & 115 & 104 & 92 & 81 & 69 & 58 & 48 & 35 & 23 & 12 & $\geq 0$ \\
\hline
\end{tabular}}

Figure 1.

Typical interface pressure maps: (a) homogenous pressure distribution, mean overall pressure 39 mmHg, and (b) heterogeneous pressure distribution concentrated in ischial region, mean overall pressure $31 \mathrm{mmHg}$. Colorbar indicates calibrated pressure in mmHg. Images oriented with back of seating surface to right $(Y)$ and left thigh to base $(X)$.

mining techniques to allow rapid maximum information recovery from the large volume of data obtained for each subject assessed. The analysis of the pressure mapping data shows that NMES is effective in changing seating interface pressures in a manner that may indicate improved tissue health. This finding has motivated our future design of an improved clinical protocol for collecting image data and developing an optimal patient-oriented NMES treatment plan. The LASR tool is available from our Web site $<$ http://stat.case.edu/lasr/> $>$. Further statistical justification and proofs are available in Wang et al. [9]. The current article uses terminology that is suitable for the clinical and biomedical communities. The clinical application and three-dimensional (3-D) processing of images are emphasized. The robustness of our segmentation method against misspecification of the signal distribution is justified here for the first time.

A major issue in both research and clinical applications of interface pressure measurement is that not only has no gold standard sensor system been identified but also no gold standard methodological approach to analyzing this type of data currently exists [10]. We hope that our approach changes this situation somewhat and sets a benchmark for future comparisons.

\section{METHODS}

\section{Clinical Problem}

The pressure mapping data we used to develop the statistical algorithm were obtained from assessment of individuals with SCI who were enrolled in a study to determine the effects of NMES on tissue health. Several variables, including interface pressure measurements, were monitored to determine both long- and short-term effects of using gluteal NMES [11-12]. Since our goal here was to present a new approach to pressure mapping, other outcomes measures were outside the focus of this article. The long-term effects of chronic use of gluteal NMES were determined by assessments of static seating pressure distribution, repeated at intervals of several months. Over this time frame, hypertrophic muscle changes produced by gluteal NMES ideally lead to reduced pressure in the ischial region, producing a smoother pressure contour if a section is taken across the 
region. Short-term changes also occurred in response to alternating left (L)/right (R) stimulation that produced weight-shifting from $\mathrm{L}$ to $\mathrm{R}$ while subjects were seated in the wheelchair. These effects were determined with realtime assessment of dynamically changing pressure distributions. In this case, increased strength of muscle contractions due to long-term use of NMES ideally leads to increased amplitude of cyclical pressure variations.

Repeated assessments were obtained for 10 individuals with SCI; 2 subjects served as controls and received no NMES between assessments. One of the two control subjects subsequently received a stimulation system that provided regular NMES of the paralyzed gluteal muscles for improvement of tissue health [3]. The nine test subjects received stimulation systems that provided regular NMES of the paralyzed gluteal muscles either for exercise or standing. Thus we had a total of 11 cases to consider for analysis: 2 control cases and 9 treatment cases from 10 subjects.

Interface pressure distribution was recorded with a $40 \times 38$ cell sensor mat (Advanced Clinical Seating System, Tekscan, Inc; Boston, Massachusetts). Each cell in the sensor mat represents an individual pressure measurement. We collected the data by scanning the subject/ support interface at 2 frames/s for a $200 \mathrm{~s}$ period while the subject sat quietly to give a 400 -frame data set (called a static data movie).

We then assessed the effects of NMES by applying dynamic weight shifting stimulation to the $\mathrm{L}$ and $\mathrm{R}$ gluteal muscles; more specifically stimulation was applied in an alternating pattern to each muscle, with one muscle (L) being stimulated for $15 \mathrm{~s}$ while the other (R) was off stimulation for the same $15 \mathrm{~s}$. The stimulation activity was then reversed ( $\mathrm{L}$ off $/ \mathrm{R}$ on), leading to a 50 percent active duty cycle for each muscle, with the $\mathrm{L} / \mathrm{R}$ muscle contractions out of phase. Interface pressures were evaluated concurrently at the same scan rate (called a dynamic data movie). NMES was then discontinued and a final static data movie was obtained while the subject sat quietly in the wheelchair. This protocol was carried out at baseline and repeated at all follow-up assessments.

\section{Description of LASR Algorithm}

Our study included a large amount of image data for relatively few subjects. More specifically, 10 subjects were seen for 3 to 6 assessments each, and 1 subject had only two assessments. At each assessment, typically three movies $\left(N_{\mathrm{m}}=3\right.$ data sets) were obtained. Each movie contained a sequence of 400 frames $\left(N_{\mathrm{f}}=400\right.$ images), and each frame contained an array of $40 \times 38$ pixels ( $N_{\mathrm{p}}=40 \times 38$ data points). Therefore, each subject had $(3 \sim 6) \times N_{\mathrm{m}} \times N_{\mathrm{f}} \times N_{\mathrm{p}}=4,320,000 \sim 8,640,000$ measurements, which is a huge number compared with the small number of subjects ( $n=10$ or 11). This type of "large- $p$-small-n" data also occurs frequently in other clinical research studies, such as functional magnetic resonance imaging (fMRI) studies and microarray data analyses. Although reporting results of a single or only a few subjects is not uncommon in biomedical engineering literature [13-14], such data pose a challenge to proper statistical analysis. The multiple comparison nature of many pixels is addressed by our LASR algorithm.

Further complexity was added to the analytical process because even though the subjects were seated carefully at each assessment, ensuring a true reproduction of a previous seating posture on each visit is often not feasible in a clinical setting. Thus, performing an adequate spatial registration is often necessary to align pressure maps obtained at different times. In assessing the effects of dynamic stimulation over time, we must also ensure that comparison is made between pressure maps obtained at the same phase of stimulation, e.g., when L gluteal NMES is on. Hence, a temporal registration is also needed to ensure two dynamic pressure movies are synchronous in their comparisons. The LASR algorithm uses a multistage procedure to sequentially address the numerous challenges from the registration to multiple comparisons, "large- $p$-small- $n$ " data, and other data analysis issues (Figure 2).

Most existing registration schemes were developed for applications to radiological images, such as CT, magnetic resonance imaging, and nuclear medicine images [15-17]. Normally, a registration procedure involves two images; one is used as the reference and the other is the floating image. Typically, either a similarity measure, such as correlation coefficient or mutual information between the reference and floating images, is used for the registration process or landmarks are identified to aid in the alignment of two images [15-19]. In our study, using either correlation or mutual information to spatially align images was not appropriate because what we need to detect are the differences at the same locations (although the correlation measure can be modified for temporal alignment as shown here). Also, our images represent pressure rather than anatomical structures as seen in radiological images, and hence the landmarks will have to be different from those for anatomical images. Indeed, 


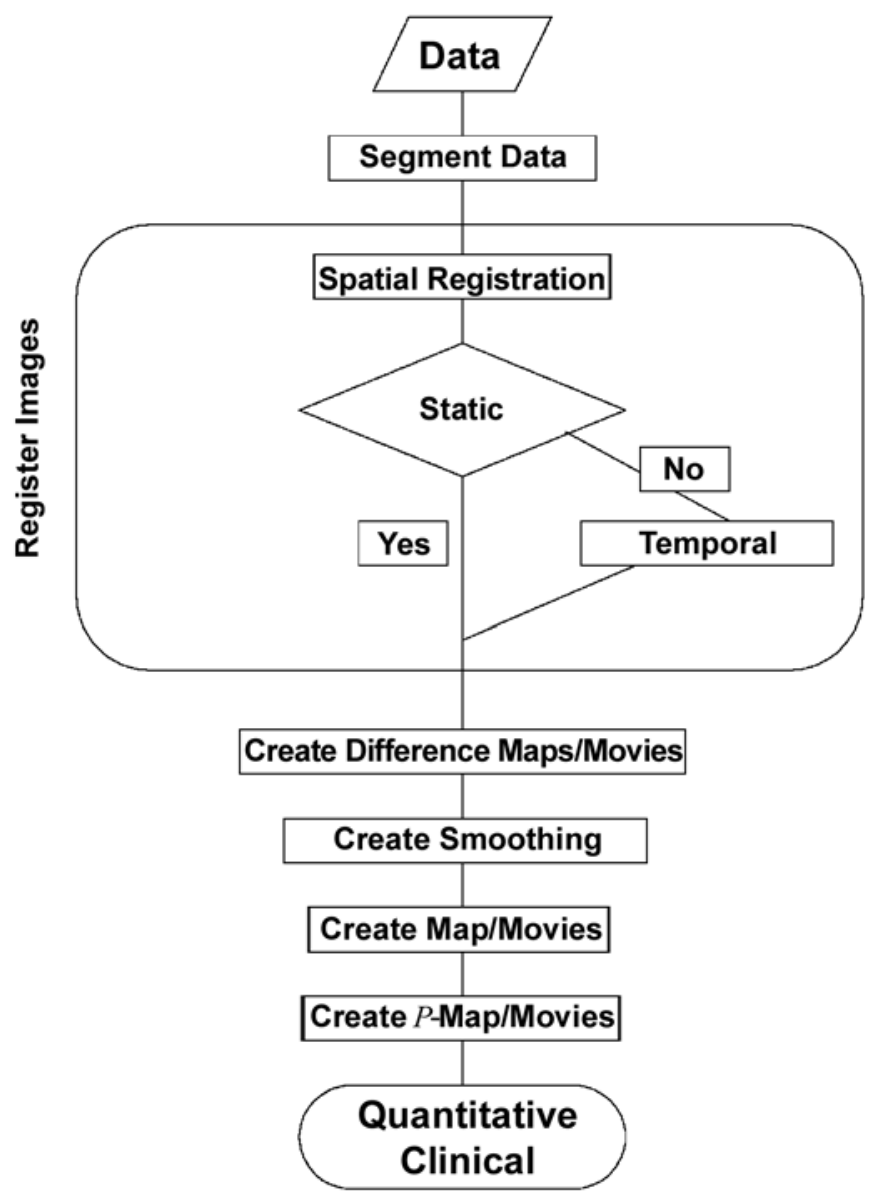

Figure 2.

Longitudinal analysis and self-registration (LASR) algorithm procedural flow chart.

the suitable landmarks for symmetrical objects such as ours are a midline (Figure 3) and an endpoint, estimated with a program based on a statistical regression analysis.

Using landmarks and registering movies frame by frame (or image by image) with a "reference" image can be done in principle but would be a daunting and highly time-consuming task in practice, thus automatic methods are important. LASR is a "self-registration" algorithm in which each image is automatically centered using its own landmarks, i.e., no reference image is needed. This selfregistration is in the same spirit as data normalization in A statistics. To the best of our knowledge, no report exists on a self-registration algorithm like ours for image registration.

In order to identify regions with significant baseline/ posttreatment differences in the NMES data, we performed pixel-by-pixel "t-type" tests (which are different
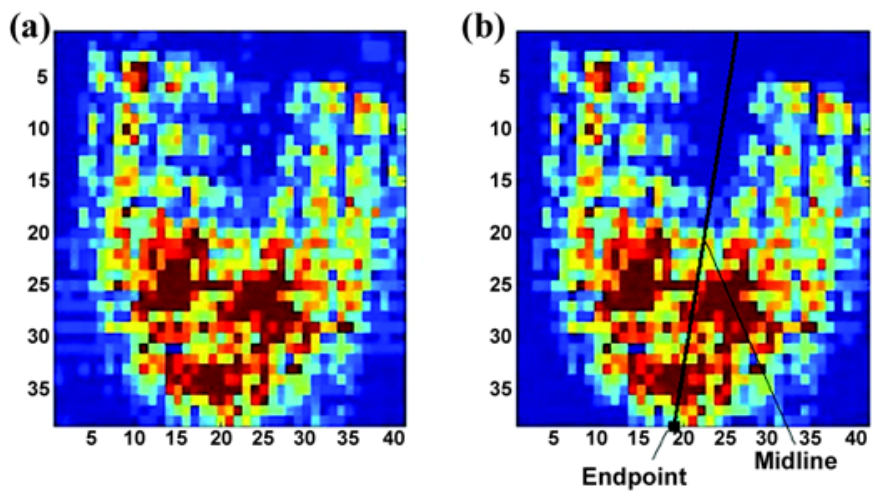

Figure 3.

Effects of segmentation on boundary properties: (a) before and (b) after segmentation. Image (b) shows location of midline, defined as midline between legs, and endpoint, defined as intersection of midline and posterior margin of region of interest. Images are oriented with back of seating surface to base and left thigh to left.

from the standard $t$-test that is based on the average of an independently and identically distributed [i.i.d.] sample) after performing bivariate local smoothing over the data. Bivariate smoothing is necessary because no replicate or i.i.d. samples are present at each pixel. This type of technique is standard in statistics and allows us to "borrow" the information from the neighborhood to mimic an i.i.d. sample. Now, the resulting $t$-type statistics at nearby pixels can be correlated. At each pixel (or data point), the null hypothesis is that no pressure difference is present between baseline and treatment. The pixels for which the test statistics exceed some threshold are classified as active or significant.

Our LASR algorithm is depicted in Figure 2 and described in detail in the Appendix (available online only at http://www.rehab.research.va.gov/), in which the robustness of the expectation maximization segmentation method is presented for the first time.

\section{LASR Summary}

The LASR output map gives a graphical representation of significant $p$-values and hence statistically significant pressure changes across the entire mapped region. The map helps us decide if gluteal NMES is effective by simultaneously comparing differences at many locations, with each pixel displaying an analogy of the simple $p$-value corrected for a false-discovery rate (FDR) threshold of no more than 0.05 . The algorithm is applied frame-by-frame 
to aligned pressure data sets. LASR maps can thus be viewed as single frame "snapshots," suitable for comparison of static seating postures or as videos for comparison of dynamically changing pressures. Application of the LASR algorithm to paired pressure data sets requires a total processing time of 2 to 3 minutes following input of the raw data to output of the LASR map.

$T$-maps show a $t$-type test statistic at each pixel and are an intermediate step in the LASR analysis procedure Appendix . T-maps derived from matched experimental data sets (from the same subjects) are preferable to those from unmatched independent experiments because nuisance factors, such as subject variation, can be reduced or subtracted in the matched experiment. Additionally, any potential effect on FDR due to possible elevated background noise is subtracted in difference images.

Since our filtering procedure is nonparametric, the outcome from our LASR procedure could be called statistical smoothing mapping in contrast to the statistical parametric mapping defined by Frackowiak et al. [20].

\section{RESULTS}

All LASR analysis is for 3-D data; however, in some cases visualizing important features from two-dimensional (2-D) images is easier. Our results are therefore presented as a combination of 2- and 3-D images to maximize the information provided. LASR analysis was applied to assess both decreases and increases in pressure from baseline. We found that for all NMES subjects, significant pressure increases were observed only in regions where no pressure would be expected, e.g., between the legs; therefore, no meaningful increases existed, and we searched only if significant decreases were noted. Because of space constraints, we present details of four typical analyses: two for control subjects and two for gluteal NMES subjects. Results for all subjects in both static and dynamic mappings are also summarized.

\section{Control Subjects}

\section{Subject A}

Seating pressure assessments were obtained at an interval of 3 months, during which time no gluteal NMES was used. Pressure mapping assessment for subject A showed some spatial misalignment (Figure 4(a)-(b)), thus spatial registration was conducted to align images before creating difference and T-maps in Figure 4(c)-(d). Qualitative evaluation of baseline/repeat pressure maps appeared, as expected, to indicate no changes in pressure distribution over time; however, statistical significance was further checked with LASR.

After applying the LASR algorithm to assess changes between baseline and repeat interface pressure data sets, we could see from the low flat T-map (Figure 4(c)-(d)) and the blank $P$-map* (not shown) that no significant differences were present in interface pressure distributions for this subject.

\section{Subject B}

Seating pressure assessments were obtained at an interval of 6 months, during which time no gluteal NMES was used. Pressure mapping assessment for subject B showed some spatial misalignment (Figure 5). Qualitative evaluation of baseline/repeat pressure maps appeared to show clearly that regional interface pressures were higher at the second assessment. This finding would appear to indicate that pressure distribution had deteriorated over time, placing the subject at higher risk of tissue breakdown. Further statistical analysis was carried out, and following registration, LASR analysis showed that these differences were not statistically significant. The FDR-controlled $P$-map is blank and is therefore not shown.

\section{Gluteal NMES Users}

\section{Static Pressure Mapping}

Subject C. At an interval of 6 months, subject C received seating pressure assessments, during which time gluteal NMES was used regularly for exercise. Pressure mapping assessment for subject $C$ appeared to show reasonable spatial alignment (Figure 6(a)-(b)), but we still conducted spatial registration to align images and correct any differences in alignment that were not visually obvious. Qualitative visual evaluation of baseline/posttreatment pressure maps also appeared to indicate some positive changes in pressure distribution over time, i.e., ischial region pressures appeared to decrease. However, this result could not be shown to be statistically significant without further detailed analysis.

\footnotetext{
*The $P$-map is the LASR output map and shows FDR-corrected probability values at each pixel. See Step 6 of Appendix.
} 

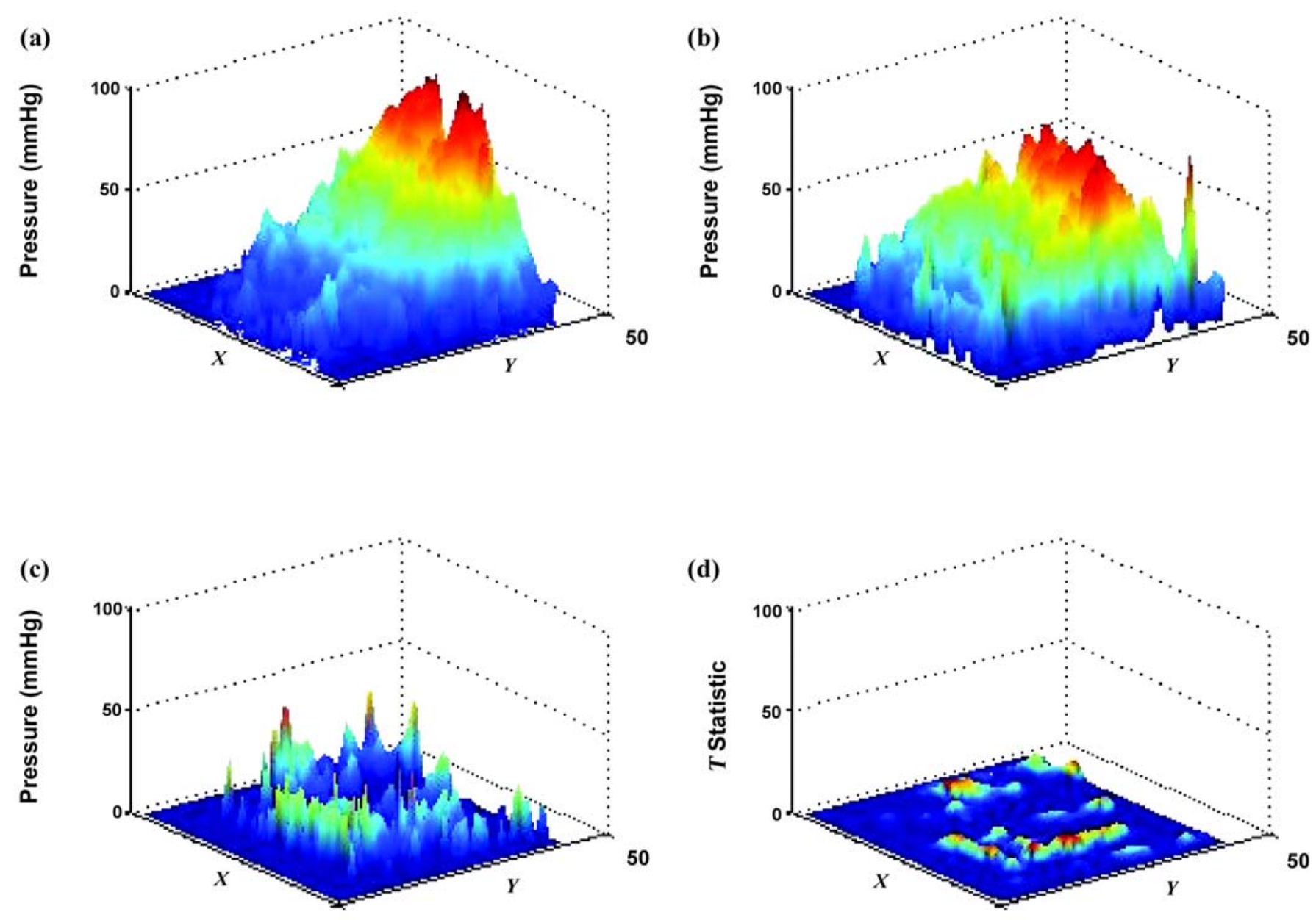

Figure 4.

Subject A assessments repeated at 3-month interval: (a) baseline control, (b) repeated measure, (c) difference after registration, (d) T-map. Subject did not use gluteal neuromuscular electrical stimulation, and no changes in treatment were noted between assessments. All images orientated with back of seating surface to right $(Y=50)$ and left thigh to base $(X=0)$. (a)-(b) Unprocessed pressure data maps; (c)-(d) longitudinal analysis and self-registration (LASR) analysis of long-term changes in static mode seated pressure distribution. (c) Difference map shows differences between baseline and last follow-up assessment after spatial alignment and registration. (d) T-map shows differences adjusted for neighborhood values, i.e., weighted average differences.

After applying the LASR analysis, we could see that some misalignment was present between images and in some regions interface pressures were reduced bilaterally over time (Figure 6(c)-(f)). Clearly, the L sacral-ischial region was more extensively affected than the $\mathrm{R}$.

Subject D. Seating pressure assessments were obtained at an interval of 6 months, during which time gluteal NMES was used regularly for exercise. Pressure mapping assessment for subject D showed poor spatial alignment, with both translation and rotation occurring between the baseline and posttreatment images (Figure 7(a)-(b)). Qualitative evaluation of longitudinal changes could not readily be performed without further image analysis. After applying the LASR algorithm to assess changes between baseline and posttreatment interface pressure data sets, we could see that pressures were reduced bilaterally over time (Figure 7(c)-(f)). The L and R sacralischial regions were equally affected.

Summary of Other Subjects. LASR algorithm outcomes are summarized for all six treatment subjects who 

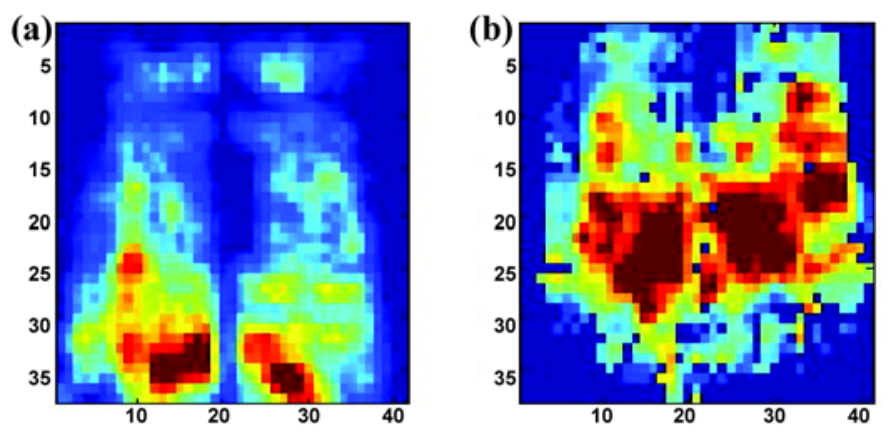

Figure 5.

Subject B assessments repeated at 6-month interval. Subject did not use gluteal neuromuscular electrical stimulation, and no changes in treatment were noted between assessments. (a) Baseline and (b) repeated measure unprocessed pressure data maps. Images are orientated with back of seating surface to base and left thigh to left.

had consistent data. Figure 8 shows 2-D $P$-maps for subjects $\mathrm{C}-\mathrm{H}$. Four of the six subjects exhibited significant changes in interface pressures in the sacral-ischial region. The extent of these changes varied from the extensive bilateral variations seen in subject $\mathrm{D}$ to small regions of change in the sacrum and $\mathrm{R}$ ischial areas of subject $\mathrm{E}$. Subject $\mathrm{E}$ also exhibited significant changes in interface pressures under the thigh region-possibly indicating a more stable sitting posture with regular use of NMES.

Subjects $\mathrm{F}$ and $\mathrm{H}$ did not show any appreciable difference in interface pressure distributions from initial to final assessment. However, baseline data were not available for these subjects and thus comparisons were made following initial conditioning.

\section{Dynamic Pressure Mapping}

In developing the temporal registration stage of the LASR algorithm, we assumed that the pressure variations exhibited a regular periodicity. This regularity allowed them to be brought into phase (temporally registered) for direct interassessment comparison. Dynamic changes in interface pressure distributions are then presented in a video format, comparable to a motion analysis output. In the current study, the effects of dynamic gluteal NMES were assessed using real-time interface pressure mapping. We proposed that the response to gluteal NMES would increase over several months of regular use as the stimulated muscles became stronger. Application of the LASR algorithm to initial stimulation data sets and response after 6 months of regular use showed significant changes in interface pressures for both subjects C and D.
Subject C showed changes predominantly on the L side under the thigh region as well as the ischial region, with some areas of change also occurring in the $\mathrm{R}$ ischial region. This result is consistent with the conclusion we had from the static movies. Subject D showed changes bilaterally in the ischial region. Relevant LASR movies can be viewed a $<\underline{\text { http://stat.case.edu/lasr/ }>\text {. }}$

\section{DISCUSSION}

The field of clinical pressure mapping has undergone great development during the past decade as a result of improved technology and graphical user interfaces. The next critical step is to improve the clinical utility of the data obtained by maximizing information retrieval.

The development of the multistage statistical LASR algorithm allows both clinicians and researchers to derive more useful, objective information from pressure maps, such as the location of significant pressure changes or the relative efficacy of pressure relief procedures. Furthermore, spatial registration allows global analysis of baseline/posttreatment differences without any subjective bias in selecting areas of interest. Further statistical justification of each component of the LASR algorithm can be found in the work of Wang and colleagues [9,21].

LASR movies rather than LASR maps can help decide which 5 percent of reported activations are most likely the false ones, because these false ones will not persistently appear to be significant over time (see difference and FDR movies on http://stat.case.edu/lasr/). As shown on our LASR Web site, those activations in the lower $\mathrm{R}$ thigh of subject $\mathrm{C}$ (upper $\mathrm{L}$ corner) and in the sacral region for subject $\mathrm{D}$ (middle $\mathrm{R}$ of image area) are most likely to be false positives. Note that for subject C, we compared the baseline session with the third session when producing both the static and dynamic data movies. For subject D, we did not have the baseline dynamic data, so the difference movie for the dynamic data is between the second and the third session, while the difference movie for the static data is between the first and third session. Nevertheless, looking at both dynamic and static $P$ movies for subject $\mathrm{D}$, we still have more information (than if we had no movies) to decide which 5 percent of reported activations might be the false ones.

In the specific study of the effects of gluteal NMES, we found that subjects who received a gluteal stimulation system showed statistically significant decreases in ischial 
(a)

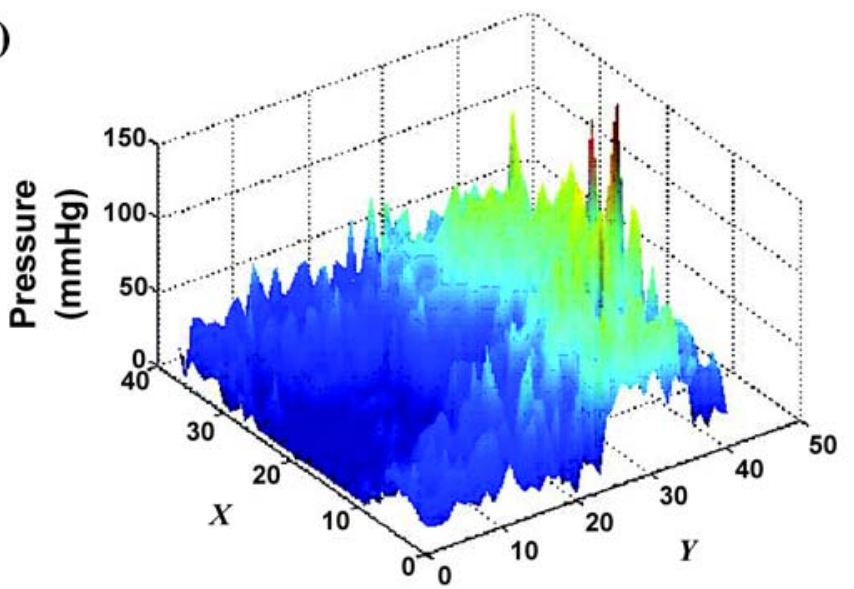

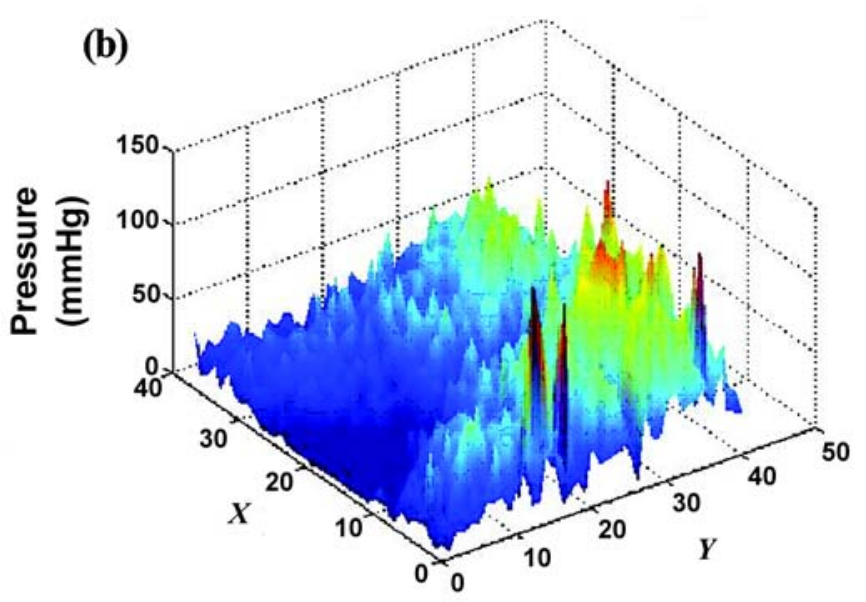

(b)

$\geq 0$ (c)

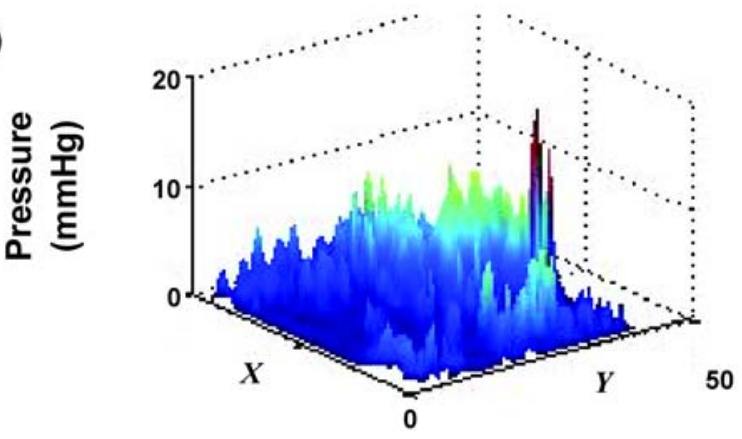

(e)

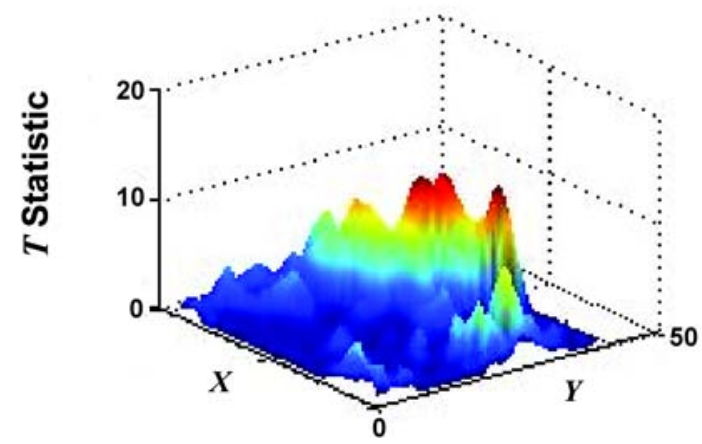

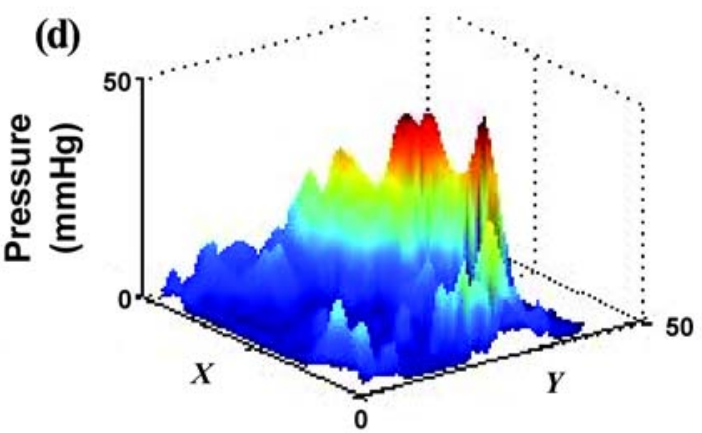

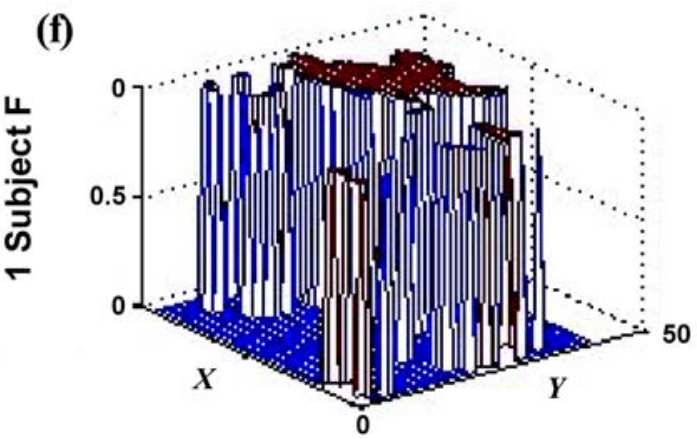

Figure 6.

Subject C assessments repeated at 6-month interval. Subject $\mathrm{C}$ used gluteal neuromuscular electrical stimulation regularly for exercise between assessments. All images are orientated with back of seating surface to right $(Y=50)$ and left thigh to base $(X=0)$. (a) Baseline and (b) repeated measure unprocessed pressure data maps, (c)-(f) Longitudinal analysis and self-registration (LASR) analysis of long-term changes in static mode seated pressure distribution. (c) Difference map after spatial alignment and registration; (d) smoothing map shows smoothed difference map; (e) $T$ map shows differences adjusted for neighborhood values, i.e., weighted average differences; (f) false-discovery rate (FDR)-controlled $P$ map shows areas of significant decrease calculated simultaneously for every data point over entire contact area. Each positive point ( $p$-value) represents point where baseline/posttreatment differences are statistically significant. This positive value is set to give pixel value of $1-p$, where $p$ is $p$-value that is < FDR cutoff value. Otherwise, pixel value is 0 . Colorbar indicates calibrated pressure in mmHg. 

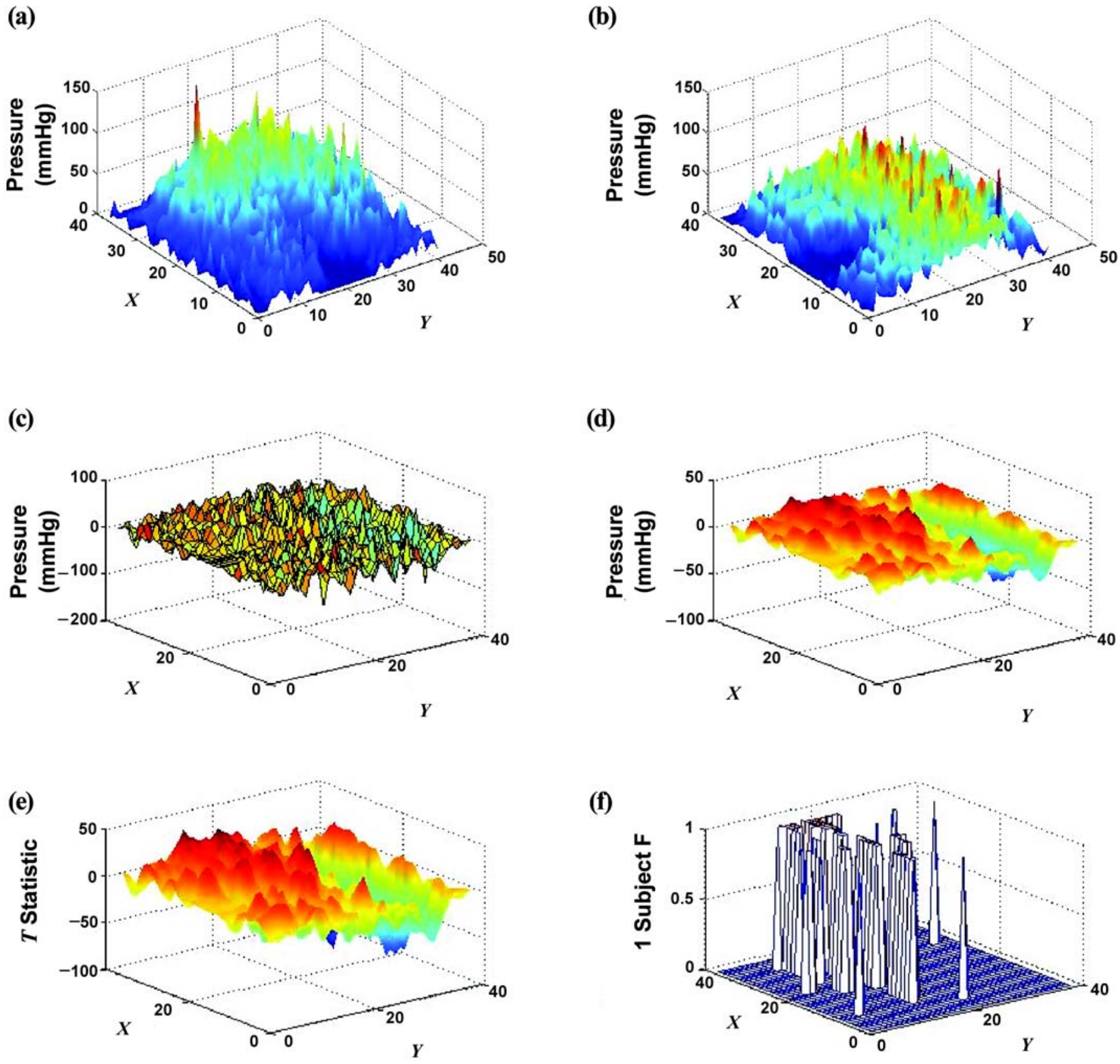

Figure 7.

Subject D assessments repeated at 6-month interval. Subject D used gluteal neuromuscular electrical stimulation regularly for exercise between assessments. (a) Baseline and (b) repeated measure unprocessed pressure data maps. Note: Pressure sensor was rotated at second assessment. Subject is seated with back of seating surface to top $(X=40)$ and left thigh to left $(Y=0)$ in baseline image. In second image, subject is seated with back of seating surface to right $(Y=50)$ and left thigh to base $(X=0)$. (c)-(f) Longitudinal analysis and self-registration (LASR) analysis of long-term changes in static mode seated pressure distribution. Images (c)-(f) are orientated with back of seating surface to top $(X=40)$ and left thigh to left $(Y=0)$. (c) Difference map after spatial alignment and registration; (d) smoothed difference map; (e) T-map shows differences adjusted for neighborhood values, i.e., weighted average differences; (f) FDR-controlled $P$-map shows areas of significant decrease calculated simultaneously for every data point over entire contact area. Each positive point ( $p$-value) represents point where baseline/posttreatment differences are statistically significant. Pixel value is calculated as for Figure 6. FDR = false-discovery rate. 

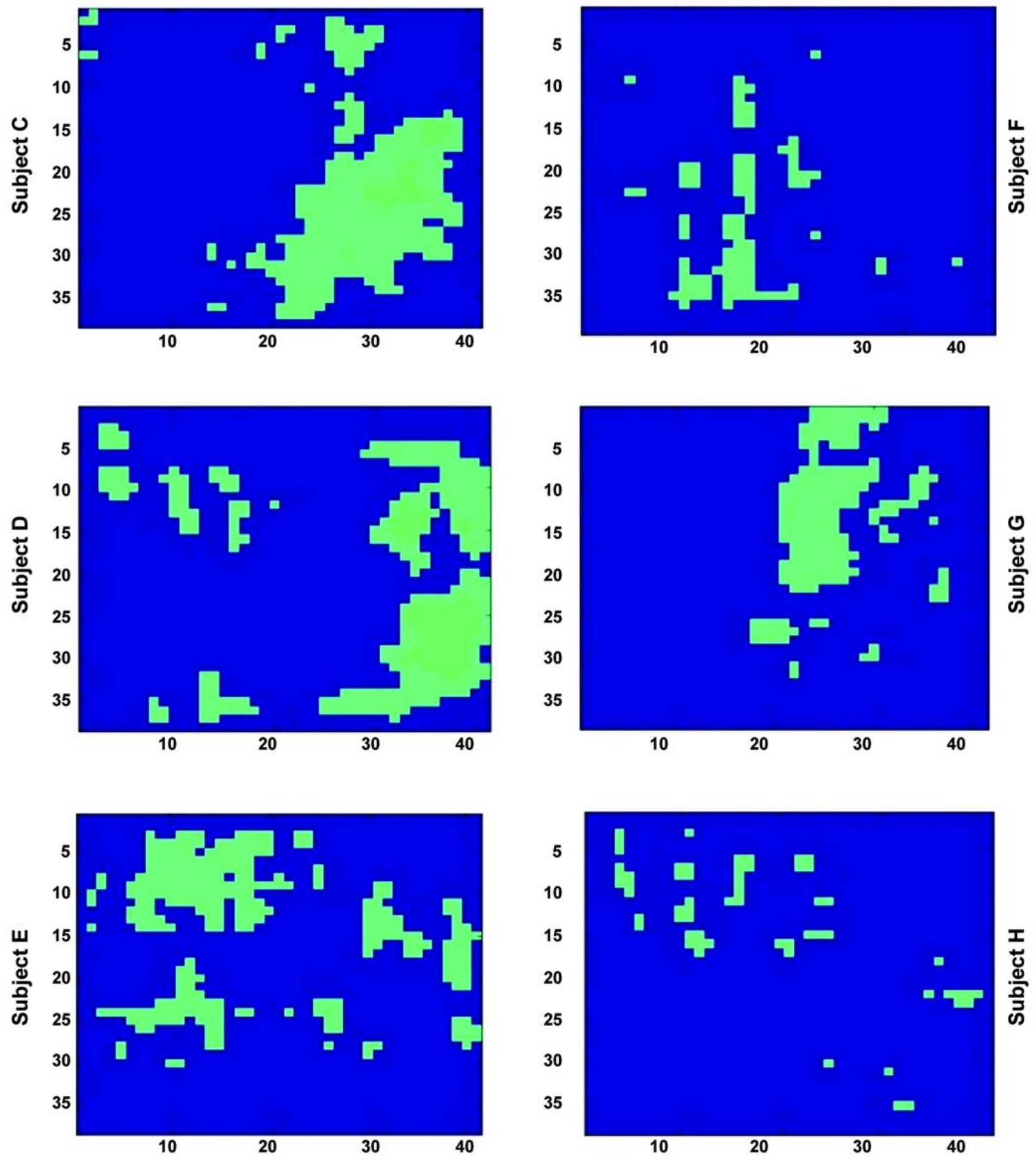

Figure 8.

Summary $P$-maps for 6 treatment subjects. All images are orientated with back of seating surface to right and left thigh to base and show points of significantly decreased pressure. 
region pressures over time when baseline/posttreatment comparisons were made. The control subject who did not receive gluteal NMES showed no significant change in seated interface pressures. We also found that statistically significant increases only occurred in regions that would not be expected to exhibit any applied pressures. This finding reinforces the need to carry out an initial measurement with no load at each assessment session in order to calibrate for variations in background brightness. This change in data collection protocol would possibly improve response detection.

The region of significant change was not symmetrical in all subjects who received gluteal NMES, reflecting the asymmetric nature both of gluteal muscle recruitment area and contractile responses. In two cases where we did not have baseline data (subjects $\mathrm{F}$ and $\mathrm{H}$ ), initial pressure distributions were obtained after initial conditioning or treatment. Comparison with data from the final assessments for these subjects did not show significant changes from the pressure distributions after the initial conditioning (Figure 8). This finding implies that perhaps for these two subjects, the majority of the intrinsic changes in tissue characteristics occurred acutely, i.e., during early treatment, and that continued regular use of gluteal NMES maintained these improved responses. Given that NMES is effective as shown in this article, this result motivates us to study the length of treatment suitable for each subject in the next phase of our study. We will also examine if what happened to these six individuals applies to other subjects after a critical time point.

A "safe" interface pressure for one individual may be a primary factor leading to tissue breakdown in another. The LASR technique determines global significant differences relative to the baseline values for each subject, with an appropriate correction for multiple comparisons. Thus changes are identified on an individualized basis rather than by a universal and somewhat arbitrary cutoff figure [4].

\section{CONCLUSIONS}

Application of the LASR algorithm enhanced data extraction from the complex pressure data sets obtained from repeated assessments of individuals using NMES for regular gluteal stimulation. We found that statistically significant decreases in the sacral-ischial region pressure distributions occurred over time. Furthermore, the dynamic response to gluteal NMES changed significantly over time with continued regular use.

Application of the LASR algorithm to our data highlighted the need to closely control reproduction of seating position between evaluations and to carry out an initial no-load measurement at each evaluation session. The need for spatial registration of pressure data can be reduced by application of a monitoring protocol to avoid severe inconsistency in future pressure mapping data.

We should also note that an analytical technique to rapidly compare 3-D images with no fixed landmarks has wide potential application. The longitudinal and spatial nature of our pressure mapping data is similar to that of fMRI data. Other clinical applications could include other soft tissue images with no bony landmarks. The experience from the current study will be helpful in the future for analysis of images from fMRI and modalities other than pressure mapping. Applications could also include situations where an imaged object changes dimensions and/or orientation over time. Nonclinical examples of this scenario include the development of insect swarms, changes in traffic patterns, or tracking ocean icebergs.

The current iteration of the LASR algorithm uses the midline as a landmark and assumes that the imaging scale is constant over time. This protocol is justified in our study; however, further development of the LASR algorithm can adapt to other types of landmarks. Advances in the LASR algorithm will encompass techniques to accommodate dimensional and periodic variability. Thus, the LASR analytical methodology has the potential to become a powerful new tool in the field of real-time image analysis.

\section{ACKNOWLEDGMENTS}

We acknowledge Scott Snyder and David Wilson for helpful discussion.

This material was based on work supported by the Rehabilitation Research and Development Service, Department of Veterans Affairs (grant B3259R to Dr. Bogie). Drs. Wang and Sun's research was supported in part by the National Science Foundation. Dr. Fei's research was partially supported by National Institutes of Health/National Cancer Institute (grant R21CA120536).

The authors have declared that no competing interests exist. 


\section{REFERENCES}

1. Crawford SA, Strain B, Gregg B, Walsh DM, PorterArmstrong AP. An investigation of the impact of the Force Sensing Array pressure mapping system on the clinical judgement of occupational therapists. Clin Rehabil. 2005; 19(2):224-31. [PMID: 15759539]

2. Sprigle S. Interface pressure measurement: Applying research findings to clinical use. In: Proceedings of the Rehabilitation Engineering and Assistive Technology Society of North America (RESNA) 26th International Conference on Technology and Diversity; 2003 Jun 19-23; Atlanta, GA. Arlington (VA): RESNA; 2003.

3. Bogie KM, Reger SI, Levine SP, Sahgal V. Electrical stimulation for pressure sore prevention and wound healing. Assist Technol. 2000;12(1):50-66. [PMID: 11067577]

4. Swain I. The measurement of interface pressure. In: Bader D, Bouten C, Colin D, Oomens C, editors. Pressure ulcer research: Current and future perspectives. Berlin (Germany): Springer; 2005. p. 51-71.

5. Brienza DM, Lin CT, Karg PE. A method for customcontoured cushion design using interface pressure measurements. IEEE Trans Rehabil Eng. 1999;7(1):99-108.

[PMID: 10188612]

6. Aissaoui R, Kauffmann C, Dansereau J, De Guise JA. Analysis of pressure distribution at the body-seat interface in able-bodied and paraplegic subjects using a deformable active contour algorithm. Med Eng Phys. 2001;23(6):359-67. [PMID: 11551812] Erratum in: Med Eng Phys. 2001; 23(10):745.

7. Hastings MK, Commean PK, Smith KE, Pilgram TK, Mueller MJ. Aligning anatomical structure from spiral X-ray computed tomography with plantar pressure data. Clin Biomech (Bristol, Avon). 2003;18(9):877-82.

[PMID: 14527816]

8. Stinson MD, Porter-Armstrong AP, Eakin PA. Pressure mapping systems: Reliability of pressure map interpretation. Clin Rehabil. 2003;17(5):504-11. [PMID: 12952156$]$

9. Wang X, Sun J, Bogie K. Spatial-temporal data mining procedure: LASR. In: Sun J, DasGupta A, Melfi V, Page C, editors. Recent developments in nonparametric inference and probability: Festschrift for Michael Woodroofe. Beachwood (OH): Institute of Mathematical Statistics; 2006. p. 213-31.

10. Remsburg RE, Bennett RG. Pressure-relieving strategies for preventing and treating pressure sores. Clin Geriatr Med. 1997;13(3):513-41. [PMID: 9227942]
11. Bogie KM, Triolo RJ. Effects of regular use of neuromuscular electrical stimulation on tissue health. J Rehabil Res Dev. 2003;40(6):469-75. [PMID: 15077659$]$

12. Bogie KM, Wang X, Triolo RJ. Long-term prevention of pressure ulcers in high-risk patients: A single case study of the use of gluteal neuromuscular electric stimulation. Arch Phys Med Rehabil. 2006;87(4):585-91. [PMID: 16571402]

13. Fei B, Duerk JL, Boll DT, Lewin JS, Wilson DL. Slice-tovolume registration and its potential application to interventional MRI-guided radio-frequency thermal ablation of prostate cancer. IEEE Trans Med Imaging. 2003;22(4): 515-25. [PMID: 12774897]

14. Wang MY, Maurer CR Jr, Fitzpatrick JM, Maciunas RJ. An automatic technique for finding and localizing externally attached markers in CT and MR volume images of the head. IEEE Trans Biomed Eng. 1996;43(6):627-37. [PMID: 8987267]

15. Little JA, Hawkes DJ. The registration of multiple medical images acquired from a single subject: Why, how, what next? Stat Methods Med Res. 1997;6(3):239-65. [PMID: 9339499]

16. Maintz JB, Viergever MA. A survey of medical image registration. Med Image Anal. 1998;2(1):1-36. [PMID: 10638851]

17. Hutton BF, Braun M. Software for image registration: Algorithms, accuracy, efficacy. Semin Nucl Med. 2003;33(3): 180-92. [PMID: 12931320]

18. Lehmann TM, Gröndahl HG, Benn DK. Computer-based registration for digital subtraction in dental radiology. Dentomaxillofac Radiol. 2001;29(6):323-46. [PMID: 11114663] Erratum in: Dentomaxillofac Radiol. 2001;30(1):64.

19. Matsopoulos GK, Delibasis KK, Mouravliansky NA, Nikita KS. Automatic medical image registration schemes using global optimization techniques. Stud Health Technol Inform. 2000;79:463-91. [PMID: 11151631]

20. Frackowiak RS, Friston KJ, Frith CD, Dolan RJ, Mazziotta JC, editors. Human brain function. San Diego (CA): Academic Press; 1997.

21. Wang $X$. New procedures for data mining and measurement error models with medical imaging applications [dissertation]. Cleveland ( $\mathrm{OH})$ : Department of Statistics, Case Western Reserve University; 2005.

Submitted for publication March 16, 2007. Accepted in revised form October 15, 2007. 